\title{
HYPERSURFACES WITH CONSTANT MEAN CURVATURE IN SPHERES
}

\author{
HILÁRIO ALENCAR AND MANFREDO DO CARMO
}

(Communicated by Peter Li)

\begin{abstract}
Let $M^{n}$ be a compact hypersurface of a sphere with constant mean curvature $H$. We introduce a tensor $\phi$, related to $H$ and to the second fundamental form, and show that if $|\phi|^{2} \leq B_{H}$, where $B_{H} \neq 0$ is a number depending only on $H$ and $n$, then either $|\phi|^{2} \equiv 0$ or $|\phi|^{2} \equiv B_{H}$. We also characterize all $M^{n}$ with $|\phi|^{2} \equiv B_{H}$.
\end{abstract}

\section{INTRODUCTION}

(1.1) Let $M^{n}$ be an $n$-dimensional orientable manifold and let $f: M^{n} \rightarrow$ $S^{n+1}(1) \subset \mathbf{R}^{n+2}$ be an immersion of $M$ into the unit $(n+1)$-sphere $S^{n+1}(1)$ of the euclidean space $\mathbf{R}^{n+2}$. Choose a unit normal field $\eta$ along $f$, and denote by $A: T_{p} M \rightarrow T_{p} M$ the linear map of the tangent space $T_{p} M$, at the point $p \in M$, associated to the second fundamental form of $f$ along $\eta$, i.e.,

$$
\langle A X, Y\rangle=\left\langle\bar{\nabla}_{X} Y, \eta\right\rangle,
$$

where $X$ and $Y$ are tangent vector fields on $M$ and $\bar{\nabla}$ is the connection of $S^{n+1}(1) . A$ is a symmetric linear map and can be diagonalized in an orthonormal basis $\left\{e_{1}, \ldots, e_{n}\right\}$ of $T_{p} M$, i.e., $A e_{i}=k_{i} e_{i}, i=1, \ldots, n$. We will denote by $H=\frac{1}{n} \sum_{i} k_{i}$ the mean curvature of $f$ and by $|A|^{2}=\sum_{i} k_{i}^{2}$.

When $f$ is minimal $(H=0)$ the following gap theorem is well known.

(1.2) Theorem. Let $M^{n}$ be compact and $f: M^{n} \rightarrow S^{n+1}(1)$ be a minimal hypersurface. Assume that $|A|^{2} \leq n$, for all $p \in M$. Then:

(i) Either $|A|^{2} \equiv 0$ (and $M^{n}$ is totally geodesic) or $|A|^{2} \equiv n$.

(ii) $|A|^{2} \equiv n$ if and only if $M^{n}$ is a Clifford torus in $S^{n+1}(1)$, i.e., $M^{n}$ is a product of spheres $S^{n_{1}}\left(r_{1}\right) \times S^{n_{2}}\left(r_{2}\right), n_{1}+n_{2}=n$, of appropriate radii.

(1.3) Remark. The sharp bound (i) is due to Simons [S]. The characterization given in (ii) was obtained independently by Chern, do Carmo, and Kobayashi [CdCK] and Lawson [L]. The result in (ii) is local.

Attempts have been made to extend the above result to hypersurfaces with constant mean curvature $H$ (see, e.g., Okumura [O]), but as far as we know no

Received by the editors January 2, 1992 and, in revised form, July 14, 1992.

1991 Mathematics Subject Classification. Primary 53C42; Secondary 53A10. tori.

Key words and phrases. Constant mean curvature, spheres, minimal surfaces, totally umbilic, 
sharp bound has yet been found. The purpose of this paper is to describe such a sharp bound and characterize the hypersurfaces that appear when the bound is reached.

For that, it is convenient to define a linear map $\phi: T_{p} M \rightarrow T_{p} M$ by

$$
\langle\phi X, Y\rangle=H\langle X, Y\rangle-\langle A X, Y\rangle .
$$

It is easily checked that trace $\phi=0$ and that

$$
|\phi|^{2}=\frac{1}{2 n} \sum_{i, j}\left(k_{i}-k_{j}\right)^{2}, \quad i, j=1, \ldots, n,
$$

so that $|\phi|^{2}=0$ if and only if $M$ is totally umbilic.

It turns out that $\phi$ is the natural object to use when extending the above theorem to constant mean curvature. In fact, Theorem 1.5 below can be proved.

We need some notation. An $H(r)$-torus in $S^{n+1}(1)$ is obtained by considering the standard immersions $S^{n-1}(r) \subset \mathbf{R}^{n}, S^{1}\left(\sqrt{1-r^{2}}\right) \subset \mathbf{R}^{2}, 0<r<1$, where the value within the parentheses denotes the radius of the corresponding sphere, and taking the product immersion $S^{n-1}(r) \times S^{1}\left(\sqrt{1-r^{2}}\right) \hookrightarrow \mathbf{R}^{n} \times \mathbf{R}^{2}$. By the choices made, the $H(r)$-torus turns out to be contained in $S^{n+1}(1)$ and has principal curvatures given, in some orientation, by

$$
k_{1}=\cdots=k_{n-1}=\frac{\sqrt{1-r^{2}}}{r}, \quad k_{n}=-\frac{r}{\sqrt{1-r^{2}}},
$$

or the symmetric of these values for the opposite orientation.

Let $M^{n}$ be compact and orientable, and let $f: M^{n} \rightarrow S^{n+1}(1)$ have constant mean curvature $H$; choose an orientation for $M$ such that $H \geq 0$. For each $H$, set

$$
P_{H}(x)=x^{2}+\frac{n(n-2)}{\sqrt{n(n-1)}} H x-n\left(H^{2}+1\right),
$$

and let $B_{H}$ be the square of the positive root of $P_{H}(x)=0$. Notice that for $H=0, B_{0}=n$.

(1.5) Theorem. Assume that $|\phi|^{2} \leq B_{H}$ for all $p \in M$. Then:

(i) Either $|\phi|^{2} \equiv 0$ (and $M$ is totally umbilic) or $|\phi|^{2} \equiv B_{H}$.

(ii) $|\phi|^{2} \equiv B_{H}$ if and only if:

(a) $H=0$ and $M^{n}$ is a Clifford torus in $S^{n+1}(1)$.

(b) $H \neq 0, n \geq 3$, and $M^{n}$ is an $H(r)$-torus with $r^{2}<\frac{n-1}{n}$.

(c) $H \neq 0, n=2$, and $M^{n}$ is an $H(r)$-torus with $r^{2} \neq \frac{n-1}{n}$.

(1.6) Remark. As it will be seen in the proof, part (ii) of Theorem (1.5) is again a local result.

(1.7) Remark. It is an interesting fact that not all $H(r)$-tori appear in the equality case for $n \geq 3$, but only those for which $r^{2}<(n-1) / n$ (it can be checked that if we orient those $H(r)$-tori for which $r^{2}>(n-1) / n$ in such a way that $H \geq 0$, then $\left.|\phi|^{2}>B_{H}\right)$. This has to do with the fact that the term which contains $H$ in the equation $P_{H}(x)=0$ vanishes when $n=2$. Thus, if $H \neq 0$, the equation defining $B_{H}$ is invariant by a change of orientation if and only if $n=2$. 
(1.8) Remark. In the minimal case, Theorem (1.2) can be extended to higher codimensions (see [CdCK]). In her doctoral dissertation of IMPA, Walcy Santos has also been able to extend Theorem (1.5) to higher codimensions (for the precise statement in this case, see $[\mathrm{Sa}])$.

\section{Proof of Theorem (1.5)}

(2.1) We first compute the Laplacian $\Delta \phi$ of $\phi$. We first observe that given a Riemannian manifold $M$ and a symmetric linear map on the tangent spaces of $M$ that satisfy formally the Codazzi equation, Cheng and Yau [CY] have already computed such a Laplacian. This turns out to be the case for $\phi$, and the result of [CY] in our context can be described as follows.

Let $\left\{e_{1}, \ldots, e_{n}\right\}$ be an orthonormal frame which diagonalizes $\phi$ at each point of $M$, i.e., $\phi e_{i}=\mu_{i} e_{i}$, and let $\nabla$ be the induced connection on $M$. Then [CY, p. 198]

$$
\frac{1}{2} \Delta|\phi|^{2}=|\nabla \phi|^{2}+\sum_{i} \mu_{i}(\operatorname{tr} \phi)_{i i}+\frac{1}{2} \sum_{i, j} R_{i j i j}\left(\mu_{i}-\mu_{j}\right)^{2},
$$

where $R_{i j i j}$ is the sectional curvature of the plane $\left\{e_{i}, e_{j}\right\}$.

We first compute the last term on the right-hand side of (2.2). By the definition of $\phi, \mu_{i}=H-k_{i}$ and, by Gauss's formula,

$$
R_{i j i j}=1+k_{i} k_{j}=1+\mu_{i} \mu_{j}-H\left(\mu_{i}+\mu_{j}\right)+H^{2} .
$$

We now use a result of Nomizu and Smyth [NS, p. 372] which implies, since $\operatorname{tr} \phi=0$, that

$$
\frac{1}{2} \sum_{i, j}\left(1+\mu_{i} \mu_{j}\right)\left(\mu_{i}-\mu_{j}\right)^{2}=n \sum_{i} \mu_{i}^{2}-\left(\sum_{i} \mu_{i}^{2}\right)^{2} .
$$

Therefore, since $\sum_{i, j}\left(\mu_{i}-\mu_{j}\right)^{2}=2 n|\phi|^{2}$, we obtain

$$
\begin{aligned}
\frac{1}{2} \sum_{i, j} R_{i j i j}\left(\mu_{i}-\mu_{j}\right)^{2}= & n \sum_{i} \mu_{i}^{2}-\left(\sum_{i} \mu_{i}^{2}\right)^{2} \\
& -\frac{H}{2} \sum_{i, j}\left(\mu_{i}+\mu_{j}\right)\left(\mu_{i}-\mu_{j}\right)^{2}+\frac{H^{2}}{2} \sum_{i, j}\left(\mu_{i}-\mu_{j}\right)^{2} \\
= & n|\phi|^{2}-|\phi|^{4}+n H^{2}|\phi|^{2}-\frac{H}{2} \sum_{i, j}\left(\mu_{i}+\mu_{j}\right)\left(\mu_{i}-\mu_{j}\right)^{2} .
\end{aligned}
$$

On the other hand, since $\sum_{i} \mu_{i}=0$, it is easily checked that

$$
\frac{1}{2} \sum_{i, j}\left(\mu_{i}+\mu_{j}\right)\left(\mu_{i}-\mu_{j}\right)^{2}=n \sum_{i} \mu_{i}^{3} .
$$

It follows from (2.3) and (2.4) that (2.2) can be written as

$$
\frac{1}{2} \Delta|\phi|^{2}=|\nabla \phi|^{2}-|\phi|^{4}+n|\phi|^{2}+n H^{2}|\phi|^{2}-n H \sum_{i} \mu_{i}^{3} .
$$

We want to estimate $\sum_{i} \mu_{i}^{3}$. For that, we use the following lemma, the inequality case of which is stated in Okumura [O]. 
(2.6) Lemma. Let $\mu_{i}, i=1, \ldots, n$, be real numbers such that $\sum_{i} \mu_{i}=0$ and $\sum_{i} \mu_{i}^{2}=\beta^{2}$, where $\beta=$ const $\geq 0$. Then

$$
-\frac{n-2}{\sqrt{n(n-1)}} \beta^{3} \leq \sum_{i} \mu_{i}^{3} \leq \frac{n-2}{\sqrt{n(n-1)}} \beta^{3},
$$

and equality holds in the right-hand (left-hand) side if and only if $(n-1)$ of the $\mu_{i}$ 's are nonpositive and equal $\left((n-1)\right.$ of the $\mu_{i}$ 's are nonnegative and equal). Proof of the lemma. We can assume that $\beta>0$, and use the method of Lagrange's multipliers to find the critical points of $g=\sum_{i} \mu_{i}^{3}$ subject to the conditions: $\sum_{i} \mu_{i}=0, \sum_{i} \mu_{i}^{2}=\beta^{2}$. It follows that the critical points are given by the values of $\mu_{i}$ that satisfy the quadratic equation

$$
\mu_{i}^{2}-\lambda \mu_{i}-\alpha=0, \quad i=1, \ldots, n .
$$

Therefore, after reenumeration if necessary, the critical points are given by:

$$
\mu_{1}=\mu_{2}=\cdots=\mu_{p}=a>0, \quad \mu_{p+1}=\mu_{p+2}=\cdots=\mu_{n}=-b<0 .
$$

Since, at the critical points,

$$
\begin{aligned}
\beta^{2} & =\sum_{i} \mu_{i}^{2}=p a^{2}+(n-p) b^{2}, \\
0 & =\sum_{i} \mu_{i}=p a-(n-p) b, \\
g & =\sum_{i} \mu_{i}^{3}=p a^{3}-(n-p) b^{3},
\end{aligned}
$$

we conclude that

$$
a^{2}=\frac{n-p}{p n} \beta^{2}, \quad b^{2}=\frac{p}{(n-p) n} \beta^{2}, \quad g=\left(\frac{n-p}{n} a-\frac{p}{n} b\right) \beta^{2} .
$$

It follows that $g$ decreases when $p$ increases. Hence $g$ reaches a maximum when $p=1$, and the maximum of $g$ is given by

$$
\begin{aligned}
a^{3}-(n-1) b^{3} & =((n-1) b)^{3}-(n-1) b^{3}=(n-2) n(n-1) b^{2} b \\
& =\frac{n-2}{\sqrt{n(n-1)}} \beta^{3} .
\end{aligned}
$$

Since $g$ is symmetric, this proves the lemma.

(2.7) Remark. For later use, it is convenient to observe from the proof that the equality holds in the right-hand side if and only if $(n-1) \mu_{i}$ 's are of the form $-b=-(1 / n(n-1))^{1 / 2} \beta$ and the remaining one is $a=((n-1) / n)^{1 / 2} \beta$.

(2.8) We return to the proof of Theorem (1.5). By using Lemma (2.6) in (2.5), we obtain

$$
\begin{aligned}
\frac{1}{2} \Delta|\phi|^{2} & \geq|\nabla \phi|^{2}-|\phi|^{4}+n\left(H^{2}+1\right)|\phi|^{2}-\frac{n(n-2)}{\sqrt{n(n-1)}} H|\phi|^{3} \\
& =|\nabla \phi|^{2}+|\phi|^{2}\left(-|\phi|^{2}-\frac{n(n-2)}{\sqrt{n(n-1)}} H|\phi|+n\left(H^{2}+1\right)\right) .
\end{aligned}
$$


Integrating both sides of the above inequality, using Stokes' theorem and the hypothesis, we conclude that

$$
0 \geq \int_{M}|\nabla \phi|^{2}+\int_{M}|\phi|^{2}\left(-|\phi|^{2}-\frac{n(n-2)}{\sqrt{(n(n-1)}} H|\phi|+n\left(H^{2}+1\right)\right) \geq 0 .
$$

Thus $|\nabla \phi|^{2} \equiv 0$ and either $|\phi|^{2} \equiv 0$ or $|\phi|^{2} \equiv B_{H}$. This proves part (i) of Theorem (1.5).

We now consider part (ii). Notice first that if $|\phi|^{2} \equiv B_{H}$, the right-hand side of inequality (2.8) vanishes identically irrespective of the compactness of $M$. Since this is all that we will use, the remaining part of the argument is local.

If $H=0$, the theorem reduces to Theorem (1.2) which gives (ii)(a).

If $H \neq 0$, we conclude that $\nabla \phi=0$ and that equality holds in the righthand side of Lemma (2.6). It follows that $k_{i}=$ const and $(n-1)$ of the $k_{i}$ 's are equal (see, e.g., [CdCK, p. 67]). After reenumeration if necessary, we can assume that

$$
k_{1}=k_{2}=\cdots=k_{n-1}, \quad k_{1} \neq k_{n}, k_{i}=\text { const } .
$$

In this situation, if $n \geq 3$, a theorem of do Carmo and Dajczer [dCD, p. 701] implies that $M^{n}$ is (contained in) a rotation hypersurfaces of $S^{n+1}(1)$ obtained by rotating a curve of constant curvature. It follows that $M^{n}$ is an $H(r)$-torus.

To identify which $H(r)$-tori do appear, we first observe that the equality case of Lemma (2.6) gives (with the enumeration above):

$$
\mu_{n}=\sqrt{\frac{n-1}{n}}|\phi|, \quad \mu_{1}=\mu_{2}=\cdots=\mu_{n-1}=-\sqrt{\frac{1}{n(n-1)}}|\phi| .
$$

Thus

$$
k_{n} k_{1}=\left(H-\sqrt{\frac{n-1}{n}}|\phi|\right)\left(H+\sqrt{\frac{1}{n(n-1)}}|\phi|\right),
$$

hence, since $|\phi|^{2} \equiv B_{H}$,

$$
n k_{n} k_{1}=n H^{2}-|\phi|^{2}-\frac{n(n-2)}{\sqrt{n(n-1)}} H|\phi|=-n,
$$

that is, $k_{n} k_{1}=-1$. On the other hand, from

$$
k_{n}=H-\mu_{n}=\frac{k_{n}+(n-1) k_{1}}{n}-\mu_{n},
$$

we conclude that

$$
(n-1) k_{n}-(n-1) k_{1}=-n \mu_{n},
$$

and, since $\mu_{n}>0$, we obtain that $k_{n}<k_{1}$. Because $k_{n} k_{1}=-1$, this implies that $k_{n}<0$. It follows that the oriented $H(r)$-torus selected by the equality case of Lemma (2.6) is given by (1.4). Since its mean curvature

$$
H=\frac{(n-1)-n r^{2}}{n r \sqrt{1-r^{2}}}
$$

is positive, we must have $r^{2}<(n-1) / n$. This completes the proof of case (b) in (ii). 
To prove finally the case (ii)(c), we observe that $M^{2} \subset S^{3}(1)$ is an isoparametric surface in $S^{3}(1)$ which is known to be either totally umbilic or an $H(r)$ torus. Since $|\phi|^{2} \neq 0, M^{2}$ is an $H(r)$-torus. By the above argument, we see that $k_{2} k_{1}=-1$. Now, however, because the equality case of Lemma (2.6) gives no additional information, we can have both cases: $k_{2}>0, k_{1}<0$ and $k_{2}<0, k_{1}>0$. Thus, the (positive) mean curvature can be either

$$
H=\frac{(n-1)-n r^{2}}{n r \sqrt{1-r^{2}}} \text { or } H=\frac{n r^{2}-(n-1)}{n r \sqrt{1-r^{2}}}, \quad n=2,
$$

and all $r^{2} \neq \frac{n-1}{n}$ will occur. This concludes the proof of (ii)(c) and of the theorem.

\section{FURTHER REMARKS}

(3.1) Theorem (1.5) raises the following question: Consider the set of hypersurfaces of $S^{n+1}(1)$ with $H=$ const and $|\phi|=$ const. Is the set of values of $|\phi|$ discrete? For minimal hypersurfaces, this question was raised in [CdCK], and even in this simple case it was shown to be a hard question. For $n=3$ and $H=0$, a significant contribution was given by Peng and Terng [PT] who showed that if $3<|\phi|^{2} \leq 6,|\phi|=$ const, then $|\phi|^{2}=6$ and $M^{3}$ is a minimal isoparametric hypersurface of $S^{4}(1)$ with three distinct principal curvatures.

The result of Peng and Terng was extended to hypersurfaces of $S^{4}(1)$ with constant mean curvature $H$ by Almeida and Brito [AB]. They proved that if $|\phi|^{2}=$ const and $|\phi|^{2} \leq 6+6 H^{2}$, then $M^{3}$ is an isoparametric hypersurface of $S^{4}(1)$ with constant mean curvature $H$; furthermore, if $4+6 H^{2} \leq|\phi|^{2} \leq$ $6+6 H^{2}$, then $|\phi|^{2}=6+6 H^{2}$ and $M^{3}$ has three distinct principal curvatures.

The result of Almeida and Brito solves the above question for $n=3$ and $|\phi|^{2} \leq 6+6 H^{2}$ and also throws some light on what happens to the $H(r)$-tori when $H \neq 0$ and $r^{2}>\frac{2}{3}$ : they are all in the interval $B_{H}<|\phi|^{2}<4+6 H^{2}$ (cf. Remark 1.7).

\section{ACKNOWLEDGMENT}

We want to thank Walcy Santos for a critical reading and the referee for valuable suggestions.

\section{REFERENCES}

[AB] S. de Almeida and F. Brito, Closed 3-dimensional hypersurfaces with constant mean curvature and constant scalar curvature, Duke Math. J. 61 (1990), 195-206.

[dCD] M. do Carmo and M. Dajczer, Rotation hypersurfaces in spaces of constant curvature, Trans. Amer. Math. Soc. 277 (1983), 685-709.

[CY] S. Y. Cheng and S. T. Yau, Hypersurfaces with constant scalar curvature, Math. Ann. 225 (1977), 195-204.

[CdCK] S. S. Chern, M. do Carmo, and S. Kobayashi, Minimal submanifolds of a sphere with second fundamental form of constant length, Functional Analysis and Related Fields (F. Browder, ed.), Springer-Verlag, Berlin, 1970, pp. 59-75.

[L] B. Lawson, Local rigidity theorems for minimal hypersurfaces, Ann. of Math. (2) 89 (1969), 187-197.

[NS] K. Nomizu and B. Smyth, A formula of Simon's type and hypersurfaces with constant mean curvature, J. Differential Geom. 3 (1969), 367-377. 
[O] M. Okumura, Hypersurfaces and a pinching problem on the second fundamental tensor, Amer. J. Math. 96 (1974), 207-213.

[PT] C. K. Peng and C. L. Terng, Minimal hypersurfaces of spheres with constant scalar curvature, Seminar on Minimal Submanifolds (E. Bombieri, ed.), Ann. of Math. Stud., no. 103, Princeton Univ. Press, Princeton, NJ, 1983, pp. 177-198.

[Sa] W. Santos, Submanifolds with parallel mean curvature vector in spheres, An. Acad. Bras. Ciênc. 64 (1992), 215-219.

[S] J. Simons, Minimal varieties in Riemannian manifolds, Ann. of Math. (2) 88 (1968), 62105.

Universidade Federal de Alagoas, Departamento de Matemática, 57072-370, Maceió, AL-BrasiL

Instituto de Matemática Pura e Aplicada, Estrada Dona Castorina 110, Jd. Botânico, 22460-320, RIO DE JANEIRO, R.J-BRASIL 